Classification

Physics Abstracts

$07.80-07.85-48.49-68.22-68.65$

\title{
Progress in the Characterization of Layered Structures by X-Ray Microanalysis
}

\author{
David G. Rickerby \\ Institute for Advanced Materials, European Commission - Joint Research Centre, 21020 Ispra (VA), \\ Italy
}

(Received March 1; accepted July 3, 1995)

\begin{abstract}
Recent advances in the mathematical description of the depth distribution of X-ray generation, $\phi(\rho z)$, have permitted the quantitative analysis of multilayer structures with a precision approaching $\pm 1 \mathrm{~nm}$. A weighted average mass absorption coefficient is proposed for use in analysis with $\mathrm{L}$ series radiation when the energy separation of the component lines is less than the spectrometer resolution. This is shown to improve agreement between experimental data and the theoretically calculated X-ray intensities. An example of the application of the $\phi(\rho z)$ method to the analysis of a titanium-boron nitride bilayer is described with special reference to the problem of detection of interfacial diffusion and oxidation phenomena.
\end{abstract}

\section{Introduction}

The development of X-ray microanalysis procedures based on a mathematical formulation of the variation of the primary ionization with mass depth, $\phi(\rho z)$, has led to substantial improvements in analytical accuracy with respect to the previous ZAF methods. This is largely due to the fact that the underlying approximation in the ZAF technique, absorption along a fixed path length related to the mean $\mathrm{X}$-ray generation depth, is replaced by a more exact integration over the entire depth range. The validity of various $\phi(\rho z)$ models has been assessed with respect to an extensive experimental data base [1-3], confirming that results are generally superior to those obtained by ZAF calculations, particularly for light element analysis.

Because of its close correspondence to physical reality, the $\phi(\rho z)$ approach can be conveniently adapted to the problem of quantitative mass thickness determination in thin films on substrates [4-6]. It is furthermore possible to extend this method to the analysis of unsupported layers [7]. The attainable depth resolution has been demonstrated to be, under favorable circumstances, comparable to that of Auger sputter profiling $[8,9]$.

Reducing the accelerating voltage of the primary electron beam increases the sensitivity to surface segregation and thin oxide or contaminant layers. The detection limit is generally $\sim$ $0.1 \mu \mathrm{g} \mathrm{cm}^{-2}$ [3]. Internal composition profiles can be determined by varying the accelerating voltage in incremental steps and comparing the measured X-ray intensities with the theoretical 
predictions for a hypothetical concentration gradient. The main limitation is that since the electron range, and hence the $\mathrm{X}$-ray generation depth, varies non-linearly with accelerating voltage, the sensitivity to segregation effects decreases with distance from the surface.

The present paper deals with the analysis of extremely thin $(\sim 10 \mathrm{~nm})$ layers deposited on silicon. It will be shown that the accuracy of the $\phi(\rho z)$ procedure can be improved by employing a weighted average mass absorption coefficient for analysis with unresolved L series lines. Factors affecting the sensitivity of the X-ray analysis technique to interdiffusion, microsegregation and oxidation effects will also be discussed.

\section{The $\phi(\rho z)$ Function}

According to the Pouchou and Pichoir (PAP) model [1, 3] the depth distribution of primary ionization can be represented by a curve consisting of two parabolic regions:

$$
\begin{gathered}
\phi_{1}(\rho z)_{0 \rightarrow R_{\mathrm{c}}}=A_{1}\left(\rho z-R_{\mathrm{m}}\right)^{2}+B_{1} \\
\phi_{2}(\rho z)_{R_{\mathrm{c}} \rightarrow R_{\mathrm{x}}}=A_{2}\left(\rho z-R_{\mathrm{x}}\right)^{2}
\end{gathered}
$$

where $R_{\mathrm{c}}$ is the mass depth at which the equations (1) and (2) are equivalent, $R_{\mathrm{m}}$ is the mass depth at which the function $\phi(\rho z)$ reaches its maximum and $R_{\mathrm{x}}$ is that at which it vanishes.

An alternative model (XPP) has been proposed by the same authors [3, 10], in which the distribution function takes the form:

$$
\phi(\rho z)=A \exp (-a \rho z)+[B \rho z+\phi(0)-A] \exp (-b \rho z)
$$

where $\phi(0)$ is the surface ionization. This expression has increased flexibility for curve fitting and is mathematically more tractable for performing calculations. It may be preferable at low overvoltages due to the difficulty of obtaining a satisfactory solution of the PAP equations.

Bastin and co-workers $[2,11]$ have suggested a different form of the $\phi(\rho z)$ function based on the Gaussian model of Packwood and Brown [12]. The calculations in the present paper employed the Pouchou and Pichoir models in the versions supplied as the STRATA ${ }^{1}$ software. The XPP model was found to give the least total error where $\mathrm{K}$ and $\mathrm{L}$ lines were included in the analysis, while the PAP procedure was generally better if only $\mathrm{K}$ lines were considered.

For a layered specimen, neglecting factors that are equal for both the specimen and standard, the emerging intensity from layer $i$ can be written

$$
I_{\text {emerg }}=\prod_{n=0}^{i-1} \exp \left[-\left(X_{n}-X\right) \rho z_{n}\right] C_{\mathrm{a}} Q_{\mathrm{j}}\left(E_{0}\right) F_{i}(X)(1+f)(1+t)
$$

where $X=\mu / \rho \operatorname{cosec} \theta, \mu / \rho$ is the mass absorption coefficient, $\theta$ is the take off angle, $C_{\mathrm{a}}$ is the concentration of element $\mathrm{a}, Q_{\mathrm{j}}\left(E_{0}\right)$ is the ionization cross section, $f$ is the characteristic and continuum fluorescence and $t$ is the increase of vacancies in level $\mathrm{j}$ due to non-radiative CosterKronig transitions. $F_{i}(X)$ is the integral

$$
F_{i}(X)=\int_{\rho z_{i}}^{\rho z_{i+1}} \phi(\rho z) \exp (-X \rho z) \mathrm{d} \rho z
$$

corresponding to the intensity emitted from the $i^{\text {th }}$ layer.

( ${ }^{1}$ ) SAMx, 4 rue Galilée, 78280 Guyancourt, France. 


\section{Average L Shell Absorption Coefficient}

The resolution of an energy dispersive spectrometer is insufficient to separate the individual components of the $\mathrm{L}$ series except for high atomic number elements. This is of little consequence if the mass absorption coefficients are similar for all L shell emissions and bulk specimens are analyzed, since the absorption will be similar in both the specimen and the standard. However, significant errors may arise in the analysis of thin films due to the difference in the relative weights of the emerging $\mathrm{L}$ lines arising from the reduced absorption path length with respect to the standard. This problem is exacerbated when the $\mathrm{L}_{2,3}$ absorption edge lies between the $\mathrm{L}_{\alpha}$ and $\mathrm{L}_{\beta}$ lines, resulting in a discontinuity in the mass absorption coefficient. The elements between $\operatorname{Sc}(Z=21)$ and $\mathrm{Ge}(Z=32)$ are affected.

To calculate the weighted average mass absorption coefficient for the $\mathrm{L}$ series it is necessary to know the ratio of the $\mathrm{L}_{3}$ emission to the total $\mathrm{L}$ shell emission. This ratio is [13]

$$
\begin{aligned}
\alpha=\frac{I_{\mathrm{L}_{1, \alpha}}}{I_{\mathrm{L}_{\text {total }}}} & =\frac{\left[N_{1}\left(f_{13}+f_{12} f_{23}\right)+N_{2} f_{23}+N_{3}\right] \omega_{3}}{N_{1} v_{1}+N_{2} v_{2}+N_{3} v_{3}} \\
v_{1} & =\omega_{1}+f_{12} \omega_{2}+\left(f_{13}+f_{12} f_{23}\right) \omega_{3} \\
v_{2} & =\omega_{2}+f_{23} \omega_{3} \\
v_{3} & =\omega_{3}
\end{aligned}
$$

where $\omega_{1}, \omega_{2}$ and $\omega_{3}$ are the fluorescence yields of the $\mathrm{L}_{1}, \mathrm{~L}_{2}$ and $\mathrm{L}_{3}$ subshells respectively, $f_{12}, f_{13}$ and $f_{23}$ are the Coster-Kronig transition probabilities and $N_{1}, N_{2}$ and $N_{3}$ are the relative numbers of primary vacancies in the $\mathrm{L}$ shell $\left(N_{1}=0.25, N_{2}=0.25, N_{3}=0.5\right)$. It follows that, to a first approximation, the average mass absorption coefficient for the $\mathrm{L}$ shell is given by

$$
(\mu / \rho)_{\mathrm{av}} \approx \alpha(\mu / \rho)_{\mathrm{L}_{\alpha}}+(1-\alpha)(\mu / \rho)_{\mathrm{L}_{\beta}}
$$

if $(\mu / \rho)_{\mathrm{L}_{1}} \simeq(\mu / \rho)_{\mathrm{L}_{\alpha}}$ and the $\mathrm{L}_{\beta}$ line constitutes the majority of the radiation emitted from subshells other that $\mathrm{L}_{3}$. The fluorescence yields and the Coster-Kronig transition probabilities are taken from tabulations of Bambynek et al. [13] of theoretical values due to McGuire [14].

The influence of the mass absorption coefficient on the emission of the $\mathrm{L}_{\alpha}$ and $\mathrm{L}_{\beta}$ lines and the total $\mathrm{L}$ shell radiation from a $10 \mathrm{~nm}$ titanium layer on a silicon substrate is shown in Figure 1 . The relative intensity of the $L_{\beta}$ line is enhanced by the virtual absence of absorption in the thin metal layer compared to a bulk standard. Therefore the total L shell $k$-ratio is higher than that of the $\mathrm{L}_{\alpha}$ emission alone.

\section{Experimental Details}

A thin bilayer consisting of $15 \mathrm{~nm}$ of boron nitride on $10 \mathrm{~nm}$ of titanium was deposited on a pure silicon substrate by means of a dual target r.f. sputtering technique [15]. The nominal thickness of the layers was confirmed by cross sectional TEM observation [8].

Energy dispersive $\mathrm{X}$-ray spectra were acquired from the as-deposited specimen at accelerating voltages of 3 and $5 \mathrm{kV}$ by a windowless $\mathrm{Si}(\mathrm{Li})$ detector with a take off angle of 35 degrees. The standards employed were pure elements, $\mathrm{Si}_{3} \mathrm{~N}_{4}$ and $\mathrm{MgO}$. A multiple least squares peak fitting routine was applied to the digitally filtered spectra to separate the $\mathrm{NK}_{\alpha}$ and TiL peaks.

The specimen was subsequently oxidized by exposure to the atmosphere at room temperature for approximately $10,000 \mathrm{~h}$. Wave dispersive spectra were acquired from the oxidized specimen 


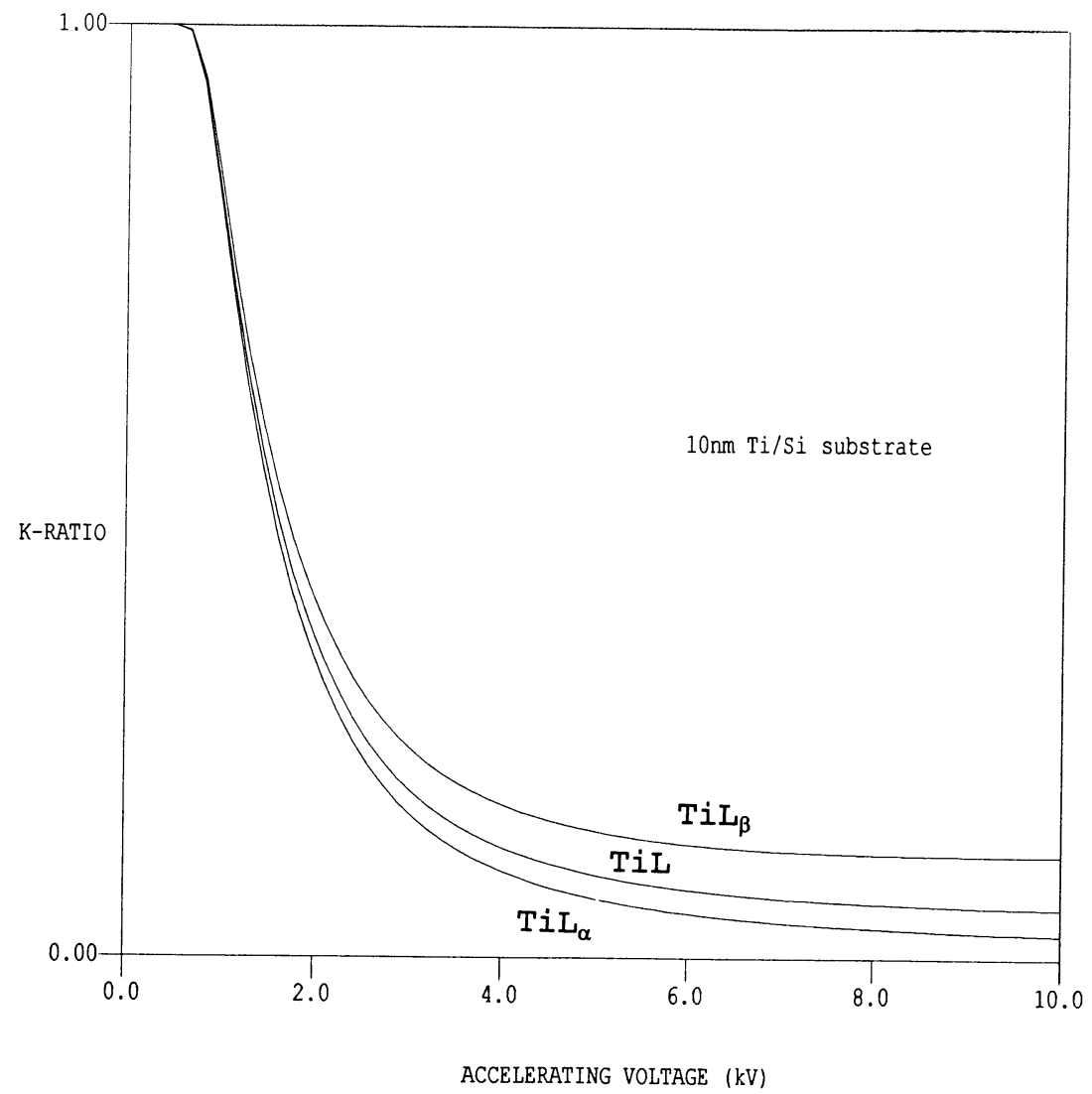

Fig. 1. - Theoretical variation of the $\mathrm{L}_{\alpha}, \mathrm{L}_{\beta}$ and total $\mathrm{L}$ emission with accelerating voltage for a $10 \mathrm{~nm}$ titanium layer on a silicon substrate.

at accelerating voltages in the range 1 to $8 \mathrm{kV}$ with a take off angle of 40 degrees. Pure elements and $\mathrm{Cr}_{2} \mathrm{~N}$ and $\mathrm{Y}_{3} \mathrm{Fe}_{5} \mathrm{O}_{12}$ were used as standards.

Calculations were performed iteratively, using the XPP model for the energy dispersive data and the PAP model for the wave dispersive data. The iteration terminated when the sum of the deviations of experimental data from the theoretically predicted curves was minimized. The mass absorption coefficients were obtained from Heinrich's MAC 30 compilation [16] or the table for ultra light elements recommended by Pouchou and Pichoir [3]. An average mass absorption coefficient for the total TiL emission was computed from equation (7).

\section{Analysis of Discrete Layers}

Experimental $k$-ratios for the as-deposited Ti-BN bilayer are compared in Figure 2 with the theoretical intensity variation predicted by optimizing the fit with the $\phi(\rho z)$ model by means of the iterative procedure. The agreement between experimental data and the theoretical curves calculated with the weighted average TiL absorption coefficient is significantly improved with respect to previous results using the $\mathrm{L}_{\alpha}$ absorption coefficient [8]. Calculations were made for two different hypotheses: i) a uniform concentration of oxygen throughout the boron nitride layer; ii) oxygen confined within a thin surface layer. Either hypothesis produced a virtually identical variation of 


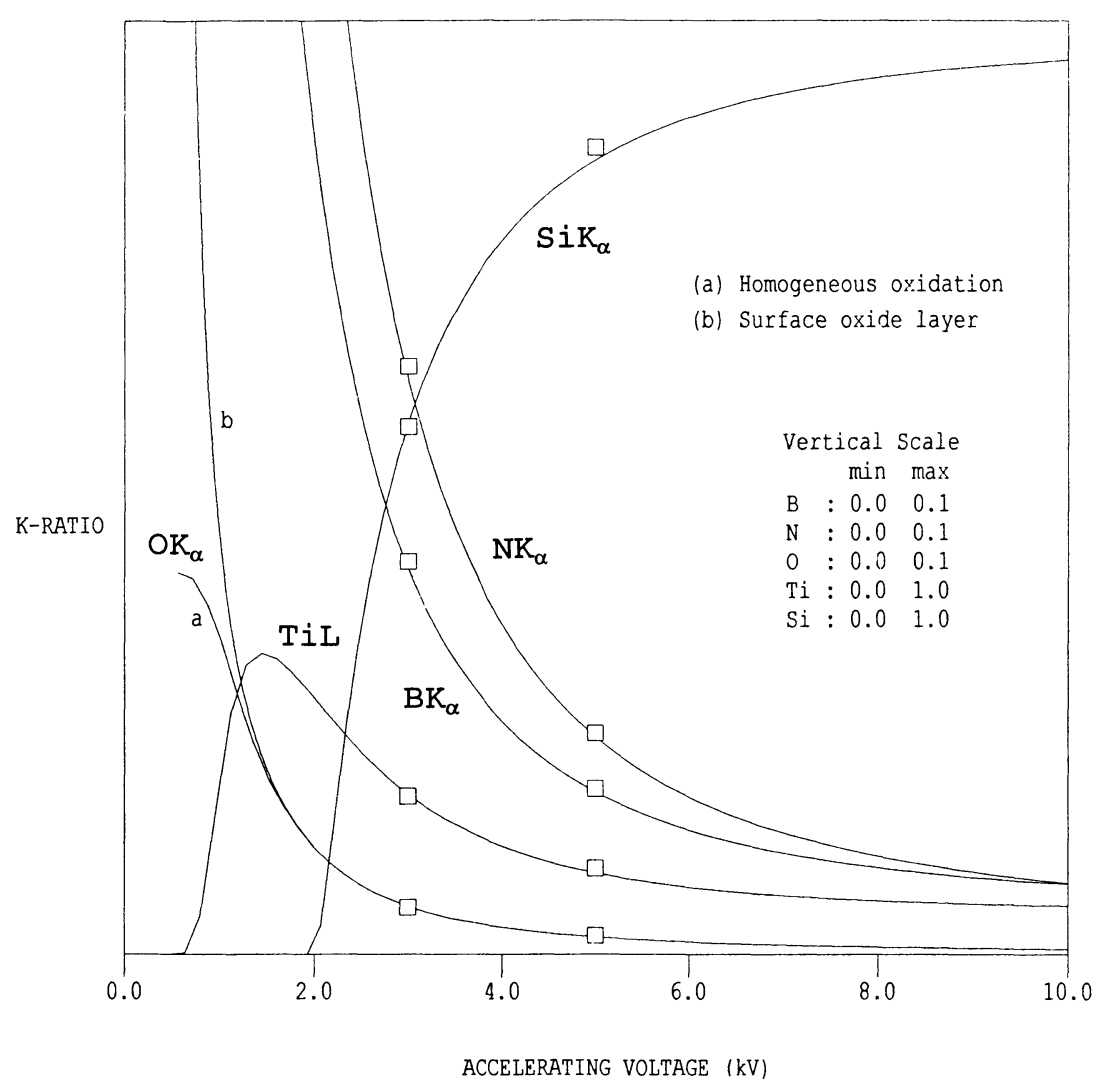

Fig. 2. - Experimental EDS intensity data for a $15 \mathrm{~nm}$ BN/10 $\mathrm{nm}$ Ti bilayer in comparison with theoretical curves fitted using the XPP model.

$\mathrm{X}$-ray intensity with accelerating voltage for elements other than oxygen. The theoretical $\mathrm{OK}_{\alpha}$ intensities diverged only at accelerating voltages less than $2 \mathrm{kV}$. To confirm the existence of a thin surface oxide layer would thus require critical experiments in the low voltage regime.

Assuming a homogeneous distribution of oxygen in the boron nitride layer, the calculated mass thickness was $2.98 \mu \mathrm{g} \mathrm{cm}^{-2}$, equivalent to $14.9 \mathrm{~nm}$ for a density of $2 \mathrm{~g} \mathrm{~cm}^{-3}$. The resulting B:N ratio corresponds to almost exact stoichiometry with a minor oxygen content of 3.5 at $\%$. A mass thickness of $4.02 \mu \mathrm{g} \mathrm{cm}^{-2}$ was calculated for the titanium layer, equivalent to $8.9 \mathrm{~nm}$ if the density is $4.5 \mathrm{~g} \mathrm{~cm}^{-3}$. The postulated surface oxide layer would have a thickness of $1.7 \mathrm{~nm}$ and an average composition corresponding approximately to $\mathrm{B}_{2} \mathrm{~N}_{1.2} \mathrm{O}_{1.8}$. The thickness of the boron nitride layer would consequently be reduced to $13.3 \mathrm{~nm}$, while that of the titanium layer would remain practically invariant. Excellent agreement between the estimated BN layer thickness and the $\mathrm{K}_{\alpha}$ intensity data, assuming a uniform oxygen concentration, is demonstrated in Figure 3. However, almost equally good agreement would be expected under the assumption of a surface oxide layer, because both hypotheses produce an identical variation of the X-ray intensity for elements other than oxygen. The TiL intensity is insensitive to the thickness of this layer, precluding a sensible comparison with experiment. 


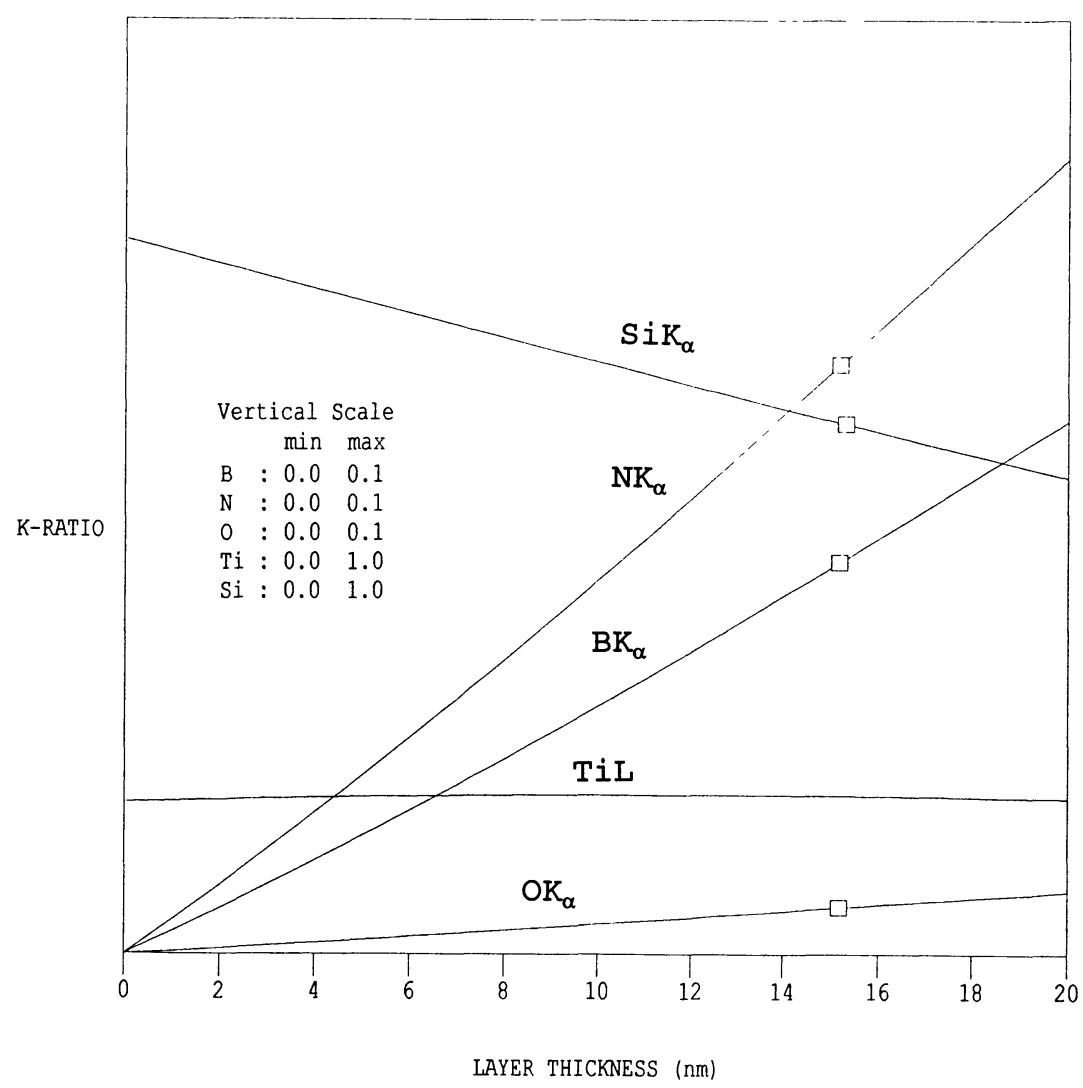

Fig. 3. - Dependence of the X-ray intensities determined at $3 \mathrm{kV}$ on the thickness of the boron nitride layer.

5.1 InTERlayer Diffusion. - Nitrogen may enter the Ti layer either due to gettering of residual gas in the vacuum chamber during deposition or by subsequent diffusion from the boron nitride overlayer. It is instructive to consider the idealized case of a uniform nitrogen concentration throughout the titanium layer, calculations for which are illustrated in Figure 4. Negligible differences in the fit were apparent up to a Ti: $\mathrm{N}$ ratio of $3: 1$, which corresponds to the limit of solid solubility for nitrogen in the $\alpha$-Ti lattice [17]. Even on increasing the Ti:N ratio to 1:1, implying transformation of the layer to stoichiometric titanium nitride, discrepancies in the emitted $\mathrm{NK}_{\alpha}$ and TiL intensities would only become evident at accelerating voltages below $2 \mathrm{kV}$. This can be regarded as a limiting case, since the formation of an interfacial TiN layer would be expected to act as a diffusion barrier.

Intermixing may also occur at the titanium-silicon interface, resulting in the formation of an amorphous layer of intermediate composition [18, 19]. Theoretical $k$-ratios were not however noticeably affected by the insertion of a Ti-Si layer of $3 \mathrm{~nm}$ thickness, equal to that measured by cross sectional TEM [8], into the layer sequence. The only significant effect was a concomitant reduction of the calculated Ti layer thickness to $7.5 \mathrm{~nm}$. If we consider the extreme case of complete intermixing (Fig. 5), differences in the TiL intensity would become apparent only below $3 \mathrm{kV}$, irrespective of the Ti:Si ratio. 


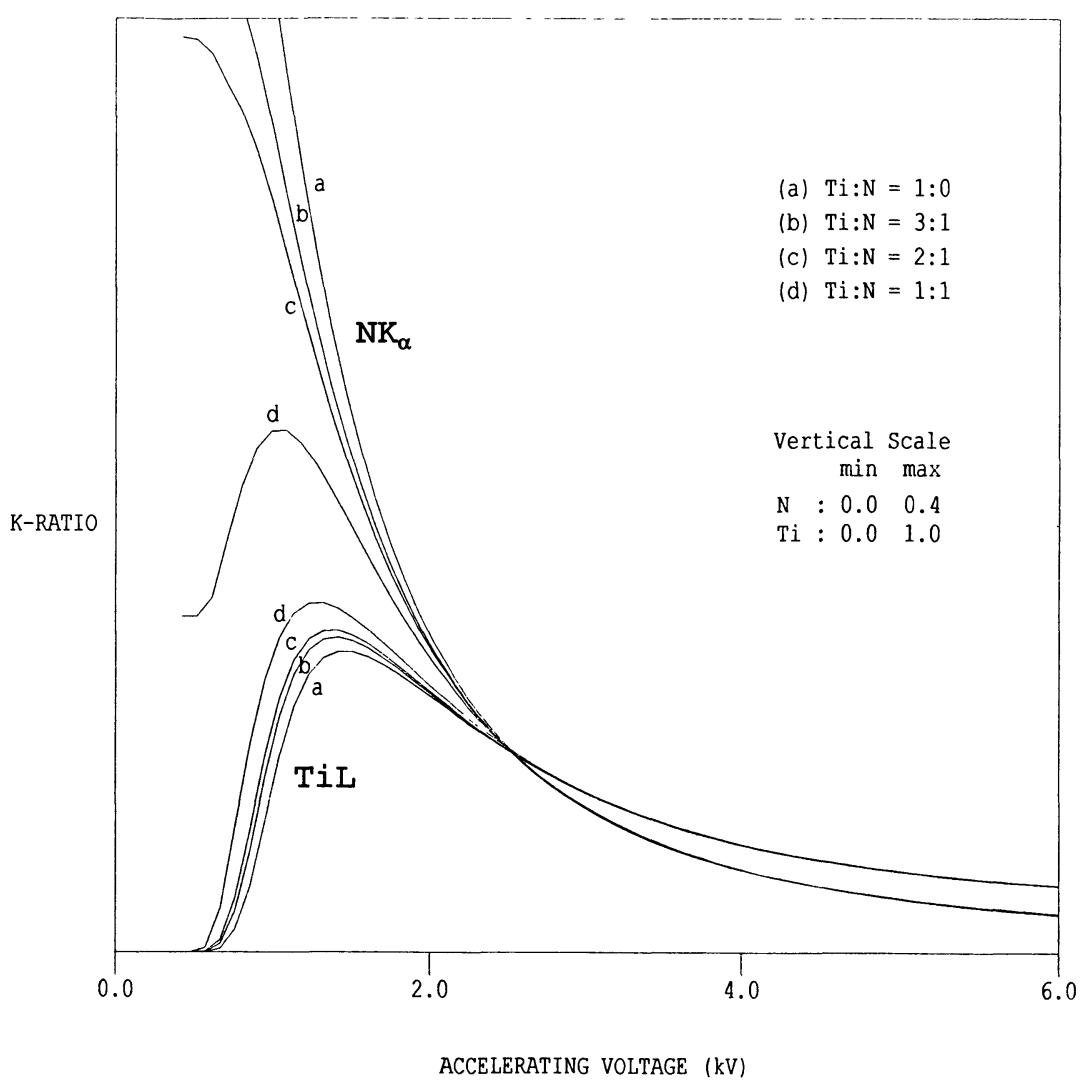

Fig. 4. - Effect of interdiffusion between the boron nitride and titanium layers on the predicted variation of $\mathrm{NK}_{\alpha}$ and TiL intensities with accelerating voltage.

5.2 Oxidation Phenomena. - To study the variation of composition with depth it is helpful to plot the experimental $k$-ratios as a function of mass thickness rather than accelerating voltage. This has been done in Figure 6 for the oxygen $\mathrm{K}_{\alpha}$ intensity data from the severely oxidized bilayer. Two different hypotheses were formulated regarding the elemental distribution within the bilayer:

i) oxygen confined to the boron nitride layer;

ii) oxygen also present in the titanium layer.

The second of these hypotheses provides the better fit to the experimental data, especially at low voltages where the $\mathrm{X}$-ray generation depth is restricted.

The composition of the oxidized boron nitride, assuming that oxygen was confined to that layer alone, was estimated as $\mathrm{B}_{3} \mathrm{~N}_{1.2} \mathrm{O}_{5.8}$, which is vastly over stoichiometric. Allowing oxygen to enter the titanium layer and optimizing the fit by trial and error, it was found that the minimum deviation was obtained at a Ti:O ratio of approximately 3:1, corresponding to the $\alpha$-Ti solid solution [17]. The resulting composition of the boron nitride layer was $\mathrm{B}_{2.1} \mathrm{~N}_{0.9} \mathrm{O}_{2}$.

This description of the structure of the oxidized bilayer is no doubt oversimplified, since concentration gradients due to interlayer diffusion [20] would also be expected to exist. Unfortunately, the lack of sensitivity of the $\phi(\rho z)$ technique to these fine scale composition changes prevents their detailed analysis. 


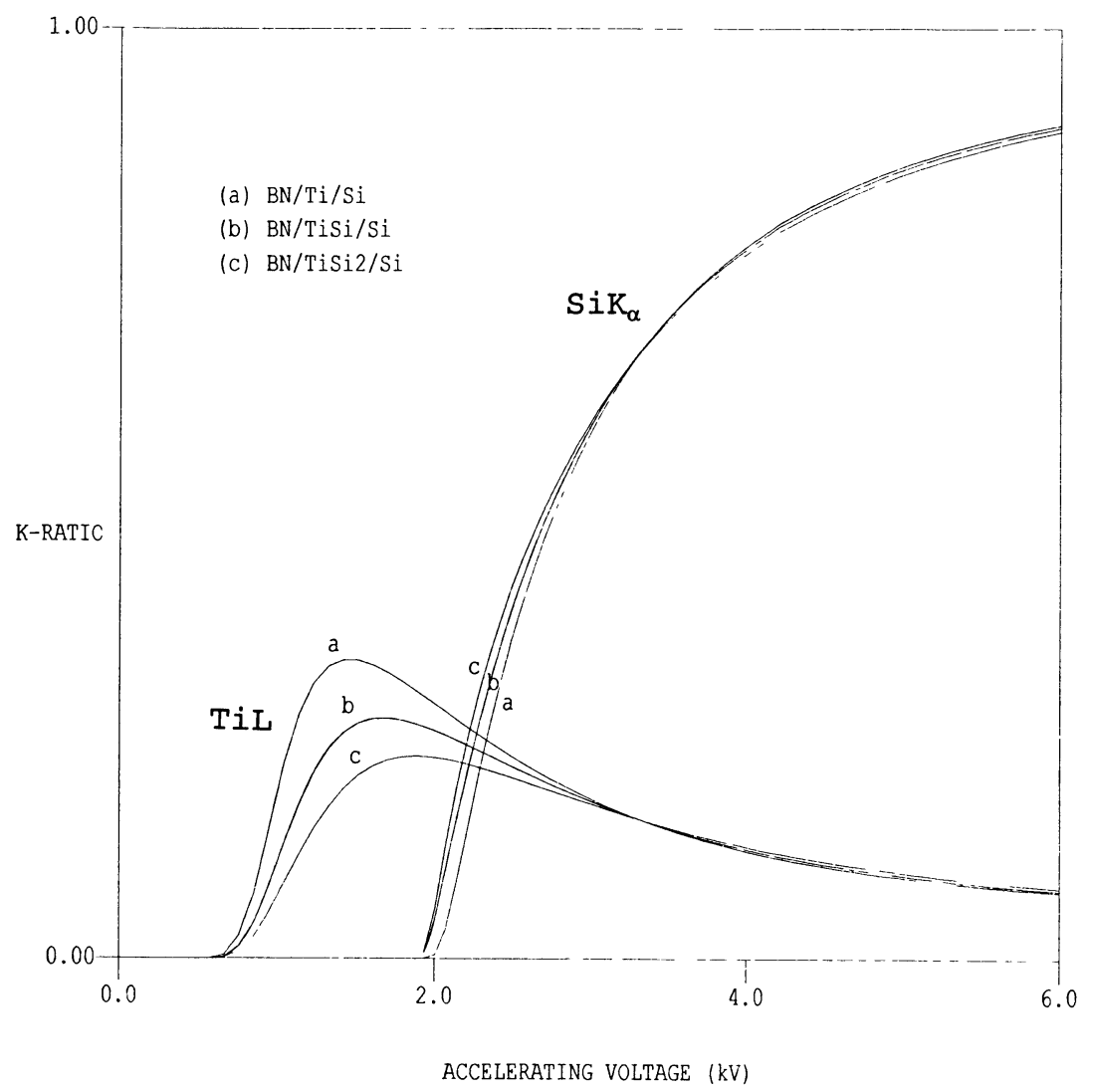

Fig. 5. - Effect of intermixing between the titanium layer and the substrate on the predicted variation of TiL and $\mathrm{SiK}_{\alpha}$ intensities with accelerating voltage.

\section{Discussion}

There is a wide variability in reported values of the mass absorption coefficients for the $\mathrm{L}_{\alpha}$ lines of the transition elements [21,22]. At least part of this uncertainty can probably be attributed to the abrupt increase in absorption in the vicinity of the $\mathrm{L}_{3}$ edge. Anomalies in absorption can also occur as a result of modification of the valence band of these elements in alloys [23]. Caution should therefore be exercised in applying relative intensity factors for $\mathrm{L}_{\alpha}$ radiation determined by wave dispersive measurements on bulk specimens [24] to analysis of thin films by energy dispersive spectroscopy.

In the present calculations it was assumed that the $\phi(\rho z)$ curve for $\mathrm{L}_{\alpha}$ radiation is valid for the entire $\mathrm{L}$ series. This is not strictly correct, because the depth distribution can be expected to be different for each subshell [25]. Substituting $\phi(\rho z)_{\mathrm{L}_{\alpha}}$ with $\phi(\rho z)_{\mathrm{L}_{\beta}}$ in the iterative procedure had, however, only an insignificant influence on the calculated mass thickness.

The adoption of a weighted average absorption coefficient for the total TiL shell emission eliminates a major source of experimental error: the difference in relative absorption of $L_{\alpha}$ and $L_{\beta}$ between the thin layer and a bulk standard. Due to the presence of the NK edge, the mass absorption coefficients for $\mathrm{Ti} \mathrm{L}_{\alpha}$ and $\mathrm{L}_{1}$ in nitrogen differ by over a factor of ten [26]. It is therefore necessary, in this case, to consider their contribution to the weighted absorption coefficient 


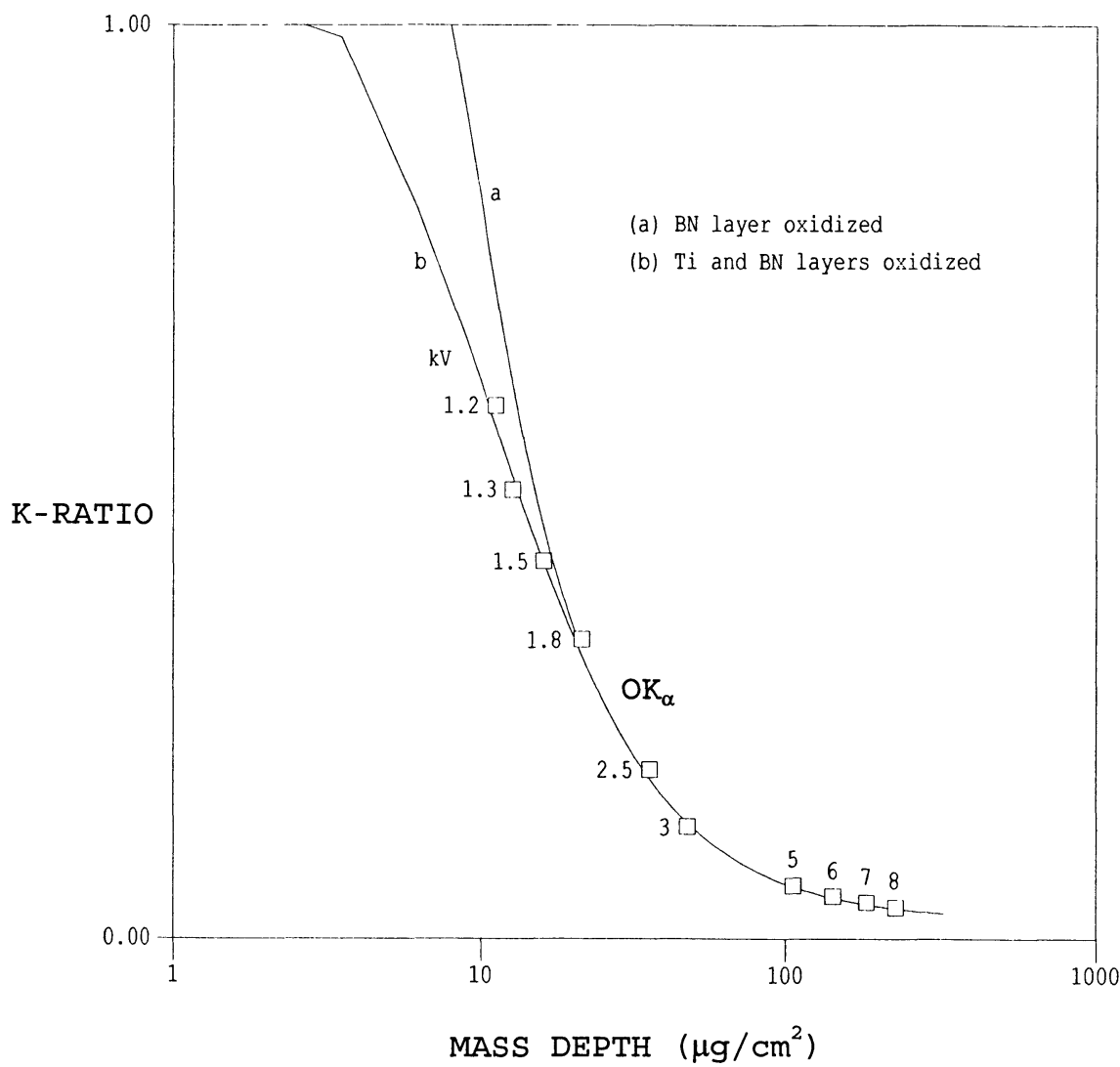

Fig. 6. - Variation of the $\mathrm{OK}_{\alpha}$ intensity with mass depth for the bilayer specimen after $10,000 \mathrm{~h}$ oxidation. Experimental WDS data have been fitted using the PAP model.

separately. Reliable theoretical calculations of the relative X-ray emission rates are available for $Z>26$, though not for all elements [27, 28]. An approximate estimate for the weighted coefficient can be made by assuming that the intensities of $\mathrm{L}_{1}$ and $\mathrm{L}_{\alpha}$ radiation emitted by pure titanium are similar [29].

Absorption in the small mass thicknesses considered here will be very slight. The emerging $\mathrm{X}$-ray intensities are not therefore greatly influenced by the depth of the radiating atom within the layer, hence the relatively poor ability to detect concentration gradients due to interdiffusion. This is most clearly evidenced by the lack of sensitivity of the TiL $k$-ratios to the thickness of an overlying boron nitride layer. It should be emphasized that these limitations are imposed by the physics of X-ray generation and absorption rather than the choice of the specific $\phi(\rho z)$ model employed.

Interdiffusion studies in Ti-TiN multilayers by cross sectional PEELS [30] have indicated that the region of intermediate composition is confined to within $\pm 3 \mathrm{~nm}$ of the interface. Since this is approximately the same order as the depth resolution for AES sputter profiling [31], it would presumably be difficult to distinguish interdiffusion of this magnitude from inherent interface broadening effects.

While the presence of a thin surface oxide layer on the as-deposited boron nitride cannot be confirmed unequivocally by the $\phi(\rho z)$ method, its estimated thickness is of similar magnitude to 
that determined for the oxide layer on a titanium nitride specimen by XPS [32]. This thickness is rather small with respect to the $\mathrm{X}$-ray generation depth even at low accelerating voltages. At $1 \mathrm{kV}$, for instance, the mass depth for X-ray emission is $\sim 10 \mu \mathrm{g} \mathrm{cm}^{-2}$, which is equivalent to several times the thickness of the entire boron nitride layer.

\section{Conclusions}

i) Use of a weighted average mass absorption coefficient improves the accuracy of the $\phi(\rho z)$ model for energy dispersive analysis with unresolved L series lines.

ii) Emitted X-ray intensities appear relatively insensitive to the effects of Ti-N and Ti-Si interdiffusion, except at very low accelerating voltages $(<3 \mathrm{kV})$.

iii) The presence of a surface oxide layer on boron nitride is practically indistinguishable from a homogeneous distribution of oxygen at low concentration. Assuming surface oxidation alone, the sensitivity of the $\phi(\rho z)$ technique can be estimated at $\sim 0.3 \mu \mathrm{g} \mathrm{cm}^{-2}$.

iv) The composition of the oxidized boron nitride layer after 10,000 $\mathrm{h}$ exposure to atmosphere represents an admixture of $\mathrm{BN}$ and $\mathrm{B}_{2} \mathrm{O}_{3}$ in approximately equal ratio.

\section{Acknowledgements}

The author is grateful to T. Friesen for assistance with thin film deposition and J.-F. Thiot for providing the wave dispersive data.

\section{References}

[1] Pouchou J.-L. and Pichoir F., J. Microsc. Spectrosc. Electron. 11 (1986) 229.

[2] Bastin G.F., Heijligers H.J.M. and van Loo F.J.J., Scanning 8 (1986) 45.

[3] Pouchou J.-L. and Pichoir F., Electron Probe Quantitation, K.F.J. Heinrich and D.E. Newbury Eds. (Plenum Press, New York, 1991) p. 31.

[4]. Pouchou J.-L. and Pichoir F., J. Phys. Colloq. France 45 (1984) C2-47.

[5] Pouchou J.-L. and Pichoir F., J. Microsc. Spectrosc. Electron. 10 (1985) 279.

[6] Bastin G.F., Dijkstra J.M., Heijligers H.J.M. and Klepper D., Mikrochim. Acta [Suppl.] 12 (1992) 93.

[7] Dijkstra J.M., Bastin G.F., Heijligers H.J.M. and Klepper D., Mikrochim. Acta 114/115 (1994) 277.

[8] Rickerby D.G. and Thiot J.-F., Mikrochim. Acta 114/115 (1994) 421.

[9] Von Richthofen A., Matsuo M., Karduck P. and Ammann N., Mikrochim. Acta 114/115 (1994) 511.

[10] Pouchou J.-L. and Pichoir F., Microbeam Analysis - 1988, D.E. Newbury Ed. (San Francisco Press, San Francisco, 1988) p. 315.

[11] Bastin G.F. and Heijligers H.J.M., Electron Probe Quantitation, K.F.J. Heinrich and D.E. Newbury Eds. (Plenum Press, New York, 1991) p. 145.

[12] Packwood R.H. and Brown J.D., X-ray Spectrom. 10 (1981) 138.

[13] Bambynek W., Bernd C., Fink R.W., Freund H.-Ü., Mark H., Swift C.D., Price R.E. and Venugopala Rao P., Rev. Mod. Phys. 44 (1972) 716.

[14] McGuire E.J., Phys. Rev. A3 (1971) 587.

[15] Gissler W., Friesen T., Haupt J. and Rickerby D.G., Surface Engineering, P.K. Datta and J.S. Gray Eds., Vol. 1 (Royal Society of Chemistry, London, 1993) p. 320. 
[16] Heinrich K.F.J., Proc. 11th Int. Cong. on X-ray Optics and Microanalysis (University of Western Ontario, 1987) p. 67.

[17] Massalski T.B., Murray J.L., Bennett L.H. and Baker H., Binary Alloy Phase Diagrams (American Society for Metals, Metals Park, 1986) p. 1652, p. 1789.

[18] Kondo I., Yoneyama T., Kondo K. and Takenaka O., J. Vac. Sci. Technol. A11 (1993) 319.

[19] Kondo I., Yoneyama T., Kondo K. and Takenaka O., Thin Solid Films 236 (1993) 236.

[20] Rickerby D.G., Philos. Mag. B68 (1993) 939.

[21] Kyser D.F., Proc. 6th Int. Cong. on X-ray Optics and Microanalysis (University of Tokyo, 1972) p. 147.

[22] Watanabe M., Horita Z. and Nemoto M., Electron Microscopy 1994, Proc. ICEM 13, Vol. 1 (Les Editions de Physique, Les Ulis, 1994) p. 605.

[23] Pouchou J.-L. and Pichoir F., J. Microsc. Spectrosc. Electron. 10 (1985) 291.

[24] Schreiber T.P. and Wims A.M., X-ray Spectrom. 11 (1982) 42.

[25] Lábár J.L., Electron Probe Quantitation, K.F.J. Heinrich and D.E. Newbury Eds. (Plenum Press, New York, 1991) p. 219.

[26] Bastin G.F., Heijligers H.J.M. and Pinxter J.F.M., Microbeam Analysis - 1988, D.E. Newbury Ed. (San Francisco Press, San Francisco, 1988) p. 290.

[27] Scofield J.H., Phys. Rev. 179 (1969) 9.

[28] Scofield J.H., Phys. Rev. A 10 (1974) 1507.

[29] Duncumb P. and Melford D.A., X-ray Optics and Microanalysis, R. Castaing, P. Deschamps and J. Philibert Eds. (Hermann, Paris, 1966) p. 240.

[30] Scott C.P., Craven A.J., Hatto P., Davies C. and Wilkinson C.D., Electron Microscopy 1994, Proc. ICEM 13, Vol. 1 (Les Editions de Physique, Les Ulis, 1994) p. 661.

[31] Zalar A., Panjan P., Kraševec V. and Hofmann S., Surf. Interface Anal. 19 (1992) 50.

[32] Bashenko O.A. and Nefedov V.I., J. Electron Spectrosc. Relat. Phenom. 53 (1990) 1.

[33] Pouchou J.-L. and Pichoir F., La Recherche Aérospatiale 5 (1984) 47. 